\title{
Grey matter atrophy in prodromal stage of dementia with Lewy bodies and Alzheimer's disease
}

\author{
Frederic Blanc ${ }^{1,2,3^{*}}$ D, Sean J. Colloby ${ }^{3}$, Benjamin Cretin ${ }^{1,2}$, Paulo Loureiro de Sousa ${ }^{2}$, Catherine Demuynck
} John T. O'Brien ${ }^{3,4}$, Catherine Martin-Hunyadi', lan McKeith' ${ }^{3}$, Nathalie Philippi ${ }^{1,2}$ and John-Paul Taylor ${ }^{3}$

\begin{abstract}
Background: Little is known about the patterns of brain atrophy in prodromal dementia with Lewy bodies (pro-DLB). Methods: In this study, we used SPM8 with diffeomorphic anatomical registration through exponentiated lie algebra to measure grey matter (GM) volume and investigate patterns of GM atrophy in pro-DLB $(n=28)$ and prodromal Alzheimer's disease (pro-AD) $(n=27)$ and compared and contrasted them with those in elderly control subjects $(n=33)$ ( $P \leq 0.05$ corrected for family-wise error).

Results: Patients with pro-DLB showed diminished GM volumes of bilateral insulae and right anterior cingulate cortex compared with control subjects. Comparison of GM volume between patients with pro-AD and control subjects showed a more extensive pattern, with volume reductions in temporal (hippocampi and superior and middle gyri), parietal and frontal structures in the former. Direct comparison of prodromal groups suggested that more atrophy was evident in the parietal lobes of patients with pro-AD than patients with pro-DLB. In patients with pro-DLB, we found that visual hallucinations were associated with relative atrophy of the left cuneus.
\end{abstract}

Conclusions: Atrophy in pro-DLB involves the insulae and anterior cingulate cortex, regions rich in von Economo neurons, which we speculate may contribute to the early clinical phenotype of pro-DLB.

Keywords: Prodromal dementia with Lewy bodies, Dementia with Lewy bodies, Alzheimer's disease, Alzheimer's dementia, Prodromal Alzheimer's disease, Lewy body disease, Mild cognitive impairment, MRI, Insula

\section{Background}

Dementia with Lewy bodies (DLB) is the second most common form of dementia after Alzheimer's disease (AD), accounting for $15-20 \%$ of neuropathologically defined cases [1]. Diagnostic classification of DLB is based on revised consensus criteria, with core diagnostic features of DLB being (1) recurrent visual hallucinations, (2) cognitive fluctuations and (3) spontaneous motor features of parkinsonism [1]. The presence of two or three of these core signs is sufficient for a diagnosis of probable DLB [1] at the stage of

\footnotetext{
* Correspondence: f.blanc@unistra.fr

${ }^{1}$ Geriatrics day hospital and neuropsychology unit. Geriatrics department and Neurology service, Memory Resources and Research Centre (CMRR),

University Hospital of Strasbourg, Strasbourg, France

${ }^{2}$ Team IMIS/Neurocrypto, French National Center for Scientific Research (CNRS), ICube Laboratory and Fédération de Médecine Translationnelle de Strasbourg (FMTS), University of Strasbourg, Strasbourg, France

Full list of author information is available at the end of the article
}

dementia, and this overlaps with the recent nomenclature update in the Diagnostic and Statistical Manual of Mental Disorders, Fifth Edition (DSM-V), where probable DLB is now comes under the heading of 'major neurocognitive disorder with Lewy bodies' [2]. In addition, DSM-V has the diagnostic classification of mild neurocognitive disorder with Lewy bodies. Thus, there is formal recognition that there is a pre-dementia state of this disease [2], similar to $\mathrm{AD}$, where there is underlying neurodegenerative disease but function has not yet been compromised. This state has also been referred to as prodromal DLB (pro-DLB), a term which we use in this paper.

Distinguishing DLB from AD at the dementia stage is difficult because of overlapping clinical and neuropathological features between the two conditions, as well as because specific symptoms of DLB, such as hallucinations or fluctuations, are not spontaneously described by 
the patient and the caregiver. At early or prodromal stages, the challenges are even greater, given the subtlety of symptoms present. Whilst cognitive patterns in proDLB have been described as different from prodromal $\mathrm{AD}$ (pro-AD) [3, 4], with patients with DLB at this early stage having more visuospatial and fluency deficits than those with $\mathrm{AD}$, and patients with $\mathrm{AD}$ having more amnesic impairments than those with DLB $[4,5]$, the neuropsychological pattern of pro-DLB appears to be more heterogeneous than that in pro-AD [5]. However, verbal memory [6] and visual memory impairments are frequent at the early stage of DLB, as in AD [7], and that is the reason why the differentiation of AD and DLB is difficult at the individual level. The behaviour modifications are also common in the two diseases: anxiety and depressive symptoms are frequent and do not permit differentiate of the two diseases [8,9].

However, accurate differentiation of DLB and AD, regardless of the stage of the disease, is important clinically, given different management trajectories for each disease (e.g., avoidance of neuroleptics in DLB, but likely better response to cholinesterase inhibitors [10]) as well as prognosis [11]. Furthermore, accurate early subtype diagnosis of the underlying neurodegenerative cause is becoming increasingly important for ensuring that future disease-modifying treatments can be targeted in individuals before substantive neurodegenerative deficits have occurred. Similarly, identification and validation of early biomarkers of pro-DLB will further assist in the development of pro-DLB criteria [12]. Specifically, for purposes of our analyses, and in alignment with our previous work [13], we consider individuals with proDLB to be those patients who meet the revised diagnostic criteria for DLB, but, instead of dementia [1], fit the criteria for mild cognitive impairment (MCI) [14].

Structural neuroimaging represents one potential biomarker area, and a well-established method is the use of voxel-based morphometry (VBM) to study atrophic change in dementia. Comparison of VBM and a manual method for hippocampal volumetry to detect temporal lobe atrophy in AD showed that VBM with or without diffeomorphic anatomical registration through exponentiated lie algebra (DARTEL) registration is equivalent or more accurate $[15,16]$. But VBM-DARTEL seems to be more accurate at detecting hippocampal atrophy in subtle cases, such as in depression [17]. This imageprocessing technique is also useful in many others neurological or psychiatric diseases including depression, schizophrenia, temporal lobe epilepsy, [18] but also MCI Parkinson's disease [19].

Previous VBM studies in DLB at the stage of dementia have demonstrated diminished volume in the insulae bilaterally and in the lateral temporal lobes, frontal lobe and precuneus [20], and others have reported grey matter (GM) volumetric reductions in the mid-brain, the substantia innominata, the hypothalamus and the right insula [21], and these findings are confirmed by a voxelwise meta-analysis on cortical atrophy of patients with DLB at the stage of dementia whose authors found bilateral insulae and basal ganglia atrophy [22]. Furthermore, this resonates with recent findings from our group. We demonstrated that the cortical thickness of patients with pro-DLB is diminished in the right anterior part of the insula [13]. This novel finding is significant, since patients at the early stage of the disease frequently have neurovegetative symptoms such as constipation, orthostatic dizziness or increased saliva, symptoms domains [23] which have been linked to insula. At the early stage, patients also frequently have cognitive fluctuations and hallucinations, and insular dysfunction has also been implicated in these symptoms [24, 25]. Contrarily, given that parkinsonism is rarely obvious at the beginning of the DLB, one might expect the putamen, pallidum and substantia nigra regions would be less structurally affected earlier in the disease [26]. However, data on subcortical structure were previously unexplored, since cortical in thickness studies researchers analysed only the cortex and not the hippocampi or basal ganglia [13].

Therefore, the primary aim of this study was to investigate cortical and subcortical grey matter (GM) atrophy patterns in patients with pro-DLB, and we report magnetic resonance imaging (MRI) patterns of GM atrophy in subjects with DLB at the stage of MCI (pro-DLB) and $\mathrm{AD}$ at the stage of MCI (pro-AD) compared with healthy elderly control subjects (HC). We hypothesised that, in pro-AD, the pattern of GM atrophy would involve predominantly the temporal lobe and parietal association cortices in keeping with the significant body of evidence supporting volumetric losses in these areas in pro-AD [27]. In contrast, we expected that the pattern of GM atrophy in pro-DLB would be less marked and more specific to structures such as the insulae.

\section{Methods}

\section{Subjects, assessments and diagnosis}

One hundred individuals suspected of having DLB or $\mathrm{AD}$ and $\mathrm{HC}$ over the age of 50 years were recruited (see Fig. 1 flowchart) from two European centres. Thirty-six were recruited from a community-dwelling population of patients referred to local old age psychiatry, geriatric medicine or neurology services in Newcastle upon Tyne (NCL); 64 were recruited from the tertiary memory clinic of Strasbourg (SXB), including neurology and geriatric medicine services. HC were recruited from established case registers. These subjects were previously included in a study of cortical thickness [13]. Subjects underwent detailed clinical and neuropsychological evaluations. Common elements between centres included 


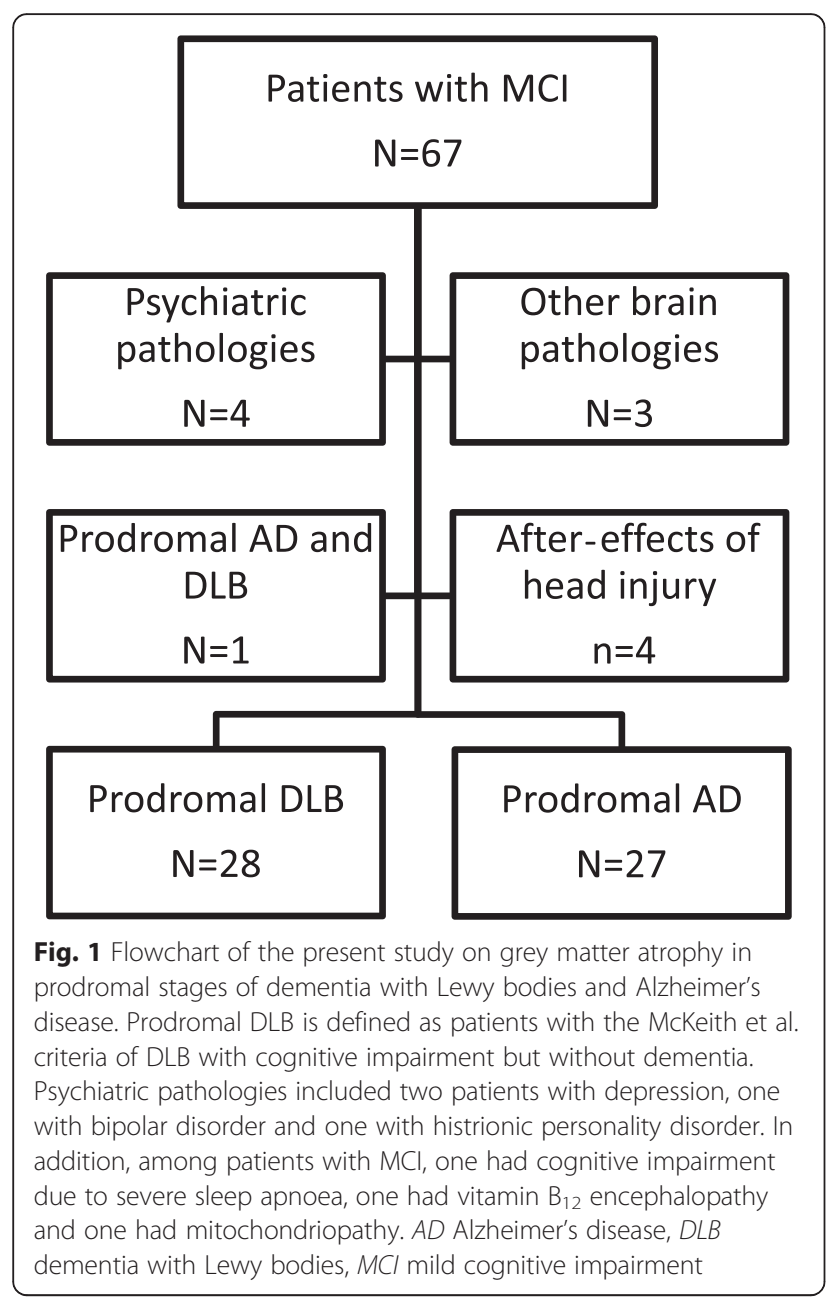

the assessment of motor parkinsonism with the Unified Parkinson's Disease Rating Scale Part III (UPDRS-III) [28], the Clinician Assessment of Fluctuation (CAF) [29], the Mini Mental State Examination (MMSE), the Clinical Dementia Rating scale (CDR) and the Trail Making Task A and B (TMTA and TMTB, respectively). For TMTA and TMTB, normative data from Tombaugh were used [30]. The neuropsychological evaluation of SXB included the Free and Cued Selective Reminding Tests for verbal memory, 48-item delayed matching to sample for visual recognition memory, forward and backward digit span, Wechsler Adult Intelligence Scale code for attention and speed processing, Frontal Assessment Battery and phonemic fluency for executive function, semantic fluency, oral denomination 80 items for language, the Rey-Osterrieth Complex Figure Test, and Mahieux praxis evaluation. The neuropsychological evaluation done at NCL was a comprehensive neuropsychological battery: the Cambridge Cognitive Examination as well as the F-A-S test and semantic fluency. For the purposes of this paper, we report only those scales which were common to both centres (e.g., MMSE, TMTA and TMTB).

Patients in the SXB group also underwent cerebrospinal fluid (CSF) analysis, including measurement of tau, phospho-tau, and amyloid- $\beta_{1-42}\left(\mathrm{~A} \beta_{1-42}\right.$; cut-off $\left.<500 \mathrm{ng} / \mathrm{L}\right)$ (INNOTEST ${ }^{\circ}$ enzyme-linked immunosorbent assay; Fujirebio Europe, Gent, Belgium). Visual assessment of medial temporal atrophy on brain MRI scans was performed using the standardised Scheltens scale (five categories, range 0-4), with 0 corresponding to no atrophy [31]. The diagnosis of neurocognitive disorder for each patient was made using the Dubois et al. criteria for pro-AD $(n=27)$ [32]. Patients with pro-DLB $(n=28)$ were defined as patients with MCI (Petersen criteria) [33] and a CDR of 0 or 0.5 , and also by the McKeith et al. criteria (meeting probable DLB criteria except presence of dementia) [1], which is consistent with recent suggestions regarding pro-DLB criteria [12]. Similarly, 33 aged healthy and cognitively intact (no MCI) subjects were recruited from among relatives and friends of subjects with neurocognitive disorders and individuals who volunteered via advertisements in local community newsletters in NCL and SXB areas. Exclusion criteria for participation in the study included contraindications for MRI, history of alcohol/ substance misuse, evidence suggesting alternative neurological or psychiatric explanations for symptoms/ cognitive impairment, focal brain lesions seen on brain imaging or the presence of other severe or unstable medical illness. All patients had formal assessment of their diagnosis done by independent expert clinicians (JPT and FB for NCL; and FB, BC and NP for SXB), and $\mathrm{HC}$ underwent clinical and cognitive assessments similar to those of patients to exclude any who may have had an occult MCI or dementia. Patients with concomitant $\mathrm{AD}$ and $\mathrm{DLB}$ (i.e., those meeting both McKeith et al. [for probable DLB] and Dubois et al. criteria) were excluded (see Fig. 1).

\section{MRI data acquisition}

Subjects in the NCL and SXB groups underwent T1weighted MRI on a 3-T MRI system within 2 months of the study assessment. NCL investigators used an 8channel head coil (Intera Achieva scanner; Philips Medical Systems, Eindhoven, The Netherlands), and SXB researchers used a 32-channel head coil (Siemens Magnetom Verio syngo MR B17; Siemens Medical Solutions, Malvern, PA, USA). The sequence obtained was a standard T1-weighted volumetric sequence covering the whole brain (3D magnetization-prepared rapid gradient echo, sagittal acquisition, 1-mm isotropic resolution). Three-dimensional (3D) T1weighted images of the NCL group had a matrix size of 240 (anterior-posterior) $\times 240$ (superior-inferior $) \times 180$ 
(right-left), a repetition time (TR) of 9.6 milliseconds, an echo time (TE) of 4.6 milliseconds and a flip angle of 8 degrees. 3D T1-weighted images of the SXB group had a matrix size of 192 (anterior-posterior) $\times 192$ (superior-inferior) $\times 176$ (right-left), a TR of 1900 milliseconds, a TE of 2.53 milliseconds and a flip angle of 9 degrees. The acquired volume was angulated such that the axial slice orientation was standardised to align with the anterior commissure-posterior commissure line.

\section{VBM-DARTEL}

Analysis was conducted using the SPM8 software package (http://www.fil.ion.ucl.ac.uk/spm) running on MATLAB 7.9 (MathWorks, Natick, MA, USA). First, MRI scans were segmented into GM, white matter (WM) and CSF using SPM8's standard unified segmentation module [34]. Second, using the DARTEL technique [35], a GM population template was derived from the entire imaging dataset (HC, pro-DLB and pro-AD). Third, after an initial affine transformation of the GM DARTEL template to the GM tissue probability map in Montreal Neurological Institute (MNI) space (http://www.mni.mcgill.ca/), non-linear warping of the segmented images was performed, matching the MNI space GM DARTEL template. Fourth, images were modulated to ensure that relative volumes of GM were preserved following spatial normalisation. Last, images were smoothed with an 8-mm full-width at halfmaximum 3D Gaussian kernel. The spatially preprocessed scans were then used for voxel-wise statistical analysis.

\section{Statistical analysis}

Controlling for age, total intracranial volume (TIV) and sex, group differences were assessed using the generalised linear model in SPM8, and statistical significance was estimated with the distributional approximations of Gaussian random fields [36]. Multiple regression analyses were also performed to investigate effects of GM loss on selected cognitive variables in pro-DLB (adjusted for age and TIV). Significant effects were identified using the corrected family-wise error (FWE) $P$ value threshold $\left(P_{\mathrm{FWE}} \leq 0.05\right)$. IBM SPSS version 22.0.0.0 software (IBM, Armonk, NY, USA) was used for further statistical evaluation as required. Where appropriate, differences in demographic and clinical data were assessed using parametric (analysis of variance [ANOVA], $t$ test) and nonparametric (Kruskal-Wallis $\mathrm{H}$ test, Mann-Whitney $U$ test) tests. In post hoc analyses, we employed Tukey's test and the Mann-Whitney $U$ test for ANOVA and Kruskal-Wallis tests, respectively. For categorical measures, $X^{2}$ tests were applied. For each test statistic, a probability value $<0.05$ was regarded as significant.

\section{Results}

\section{Subject characteristics}

The demographic data for patients and HC are summarised in Table 1. Subject groups were well matched for education, sex and handedness. Of the patients with pro-AD, 9 presented with an amnestic MCI single domain and 18 with amnestic MCI multiple domains. Of the patients with pro-DLB, 2 presented with amnestic MCI multiple domains, 13 with a non-amnestic MCI single domain and 13 with non-amnestic MCI multiple domains. For pro-AD and pro-DLB, MMSE and CDR scores were similar. For TMTA and TMTB, patients with pro-DLB were more impaired than the HC group. Patients with pro-DLB were more likely to have hallucinations and had higher motor parkinsonism (UPDRS III scores) and CAF scores. They also had a higher prevalence of rapid eye movement sleep behaviour disorder (RBD) than the prevalence in the other groups. Patients with pro-DLB were on dopaminergic treatment and had the highest use of neuroleptics (clozapine and quetiapine) compared with other groups. Patients with pro-AD had a greater number of abnormal CSF biomarkers than patients with pro-DLB. Among 23 patients with proDLB, only one had an abnormal level $A \beta_{1-42}$. Upon visual rating of hippocampal atrophy on MRI scans, we found that patients with pro-AD had more atrophy than HC. Patients with pro-DLB had more atrophy than HC only for the left hippocampus. Patients with pro-AD had more hippocampal atrophy than patients with pro-DLB only for the right hippocampus.

\section{Voxel-based morphometry group effects}

VBM analysis revealed, relative to $\mathrm{HC}$, significant GM volume loss in pro-DLB bilaterally in the insula, precuneus and medial frontal structures, as well as in the left anterior cingulate, left middle frontal, right superior and inferior frontal regions (Fig. 2a). Table 2 depicts the location and peak significance of these areas. No GM volume losses were apparent in $\mathrm{HC}$ that were greater than those in pro-DLB.

A more extensive atrophic pattern was apparent in pro-AD compared with $\mathrm{HC}$, showing significant bilateral GM loss in the hippocampal, frontal (inferior, medial, superior gyri) and middle temporal gyrus, as well as in the posterior and mid-cingulate regions (Fig. 2b). Other unilateral areas included the cerebellum, precuneus, inferior occipital, and middle frontal and superior parietal regions. Table 2 shows the location and peak significance of the regions. No GM deficits were observed in $\mathrm{HC}$ which exceeded those in pro-AD.

Between patient groups, differences in GM atrophy were much decreased and confined to the right superior parietal region in pro-AD relative to pro-DLB (Fig. 2c). Table 2 presents the location and peak 
Table 1 Clinical and demographic features of patients with dementia with Lewy bodies patients, Alzheimer's disease at the mild cognitive impairment or prodromal stage, and healthy elderly control subjects

\begin{tabular}{|c|c|c|c|c|c|}
\hline & Pro-DLB $(n=28 ; 2 \mathrm{NCL}, 26 \mathrm{SXB})$ & Pro-AD $(n=27 ; 1 \mathrm{NCL}, 26 \mathrm{SXB})$ & $\mathrm{HC}(n=33 ; 30 \mathrm{NCL}, 3 \mathrm{SXB})$ & Test statistic, $P$ value & Post hoc analysis ${ }^{\mathrm{a}}$ \\
\hline Age, years, mean (SD) & $67.5(9.2)$ & $69.3(7.8)$ & $72.4(10.4)$ & $F=2.189, P=0.118$ & \\
\hline Education, ${ }^{\mathrm{b}}$ 1/2/3 & $11 / 4 / 8$ & $11 / 3 / 12$ & $1 / 14 / 11$ & $H=2.116, P=0.347$ & \\
\hline Sex, F/M & $16 / 12$ & $7 / 20$ & $18 / 15$ & $X^{2}=6.726, P=0.035^{c}$ & \\
\hline Handedness, R/L & $26 / 2$ & $24 / 3$ & $29 / 4$ & $X^{2}=1.558, P=0.459$ & \\
\hline MMSE score & $27.6(2.1)$ & $26.9(1.9)$ & $29.4(0.9)$ & $H=31.897, P<0.0001^{c}$ & $\mathrm{HC}>$ pro- $\mathrm{AD}$ and pro-DLB \\
\hline TMTA, ${ }^{d}$ impaired subjects & $60.7 \%$ & $32.0 \%$ & $0 \%$ & $H=12.174, P<0.002^{c}$ & $H C>$ pro-DLB \\
\hline TMTB, ${ }^{d}$ impaired subjects & $71.4 \%$ & $44.0 \%$ & $0 \%$ & $H=15.245, P<0.0001^{c}$ & $\mathrm{HC}>$ pro-DLB \\
\hline CDR Sum of Boxes, 0/0.5/1/2/3 & $2 / 26 / 0 / 0 / 0$ & $1 / 26 / 0 / 0 / 0$ & $33 / 0 / 0 / 0 / 0$ & $H=75.466, P<0.0001^{c}$ & $\mathrm{HC}<$ pro-DLB and pro-AD \\
\hline \multicolumn{6}{|l|}{ Parkinsonisme } \\
\hline Rigidity, 0/1/2/3/4 & $7 / 20 / 1 / 0 / 0$ & $23 / 4 / 0 / 0 / 0$ & $33 / 0 / 0 / 0 / 0$ & $H=44.388, P<0.0001^{c}$ & Pro-DLB $>\mathrm{HC}$ and pro-AD \\
\hline Akinesia, 0/1/2/3/4 & 10/14/3/1/0 & 23/4/0/0/0 & $31 / 2 / 0 / 0 / 0$ & $H=29.156, P<0.0001^{c}$ & Pro-DLB $>\mathrm{HC}$ and pro-AD \\
\hline Tremor at rest, 0/1/2/3/4 & $17 / 9 / 2 / 0 / 0$ & $27 / 0 / 0 / 0 / 0$ & $33 / 0 / 0 / 0 / 0$ & $H=19.360, P<0.0001^{c}$ & Pro-DLB $>\mathrm{HC}$ and pro-AD \\
\hline Hallucinations & $60.7 \%$ & $0 \%$ & $0 \%$ & $X^{2}=44.521, P<0.0001^{c}$ & \\
\hline Fluctuations & $92.9 \%$ & $0 \%$ & $0 \%$ & $X^{2}=65.972, P<0.0001^{c}$ & \\
\hline $\mathrm{CAF}^{\mathrm{b}}$ & $3.5(3.6)$ & $0.0(0)$ & $0(0)$ & $\mathrm{H}=34.872, P<0.0001^{c}$ & Pro-DLB $>\mathrm{HC}$ and pro-AD \\
\hline RBD & $56.0 \%$ & $7.7 \%$ & $0 \%$ & $\mathrm{H}=31.696, P<0.0001^{c}$ & Pro-DLB $>\mathrm{HC}$ and pro-AD \\
\hline \multicolumn{6}{|l|}{ Treatment } \\
\hline ChEl & $28.6 \%$ & $48.1 \%$ & $0.0 \%$ & $X^{2}=18.253, P<0.0001^{c}$ & \\
\hline Dopa & $28.6 \%$ & $0.0 \%$ & $0.0 \%$ & $X^{2}=19.274, P<0.0001^{c}$ & \\
\hline NL & $10.7 \%$ & $0.0 \%$ & $0.0 \%$ & $x^{2}=6.793, P=0.033^{c}$ & \\
\hline \multicolumn{6}{|c|}{ CSF, mean (SD), number of subjects } \\
\hline$A \beta_{1-42}$ & $859.3(336.7) n=23$ & $579.0(287.4), n=23$ & - & $\mathrm{F}=5.345, P=0.008^{c}$ & Pro-DLB > pro-AD \\
\hline Phospho-tau & $43.6(13.9), n=23$ & $93.7(36.8), n=23$ & - & $\mathrm{F}=18.805, P<0.0001^{\mathrm{C}}$ & Pro-DLB $<$ pro-AD \\
\hline Tau & $313.0(286.3), n=23$ & $660.6(355.4), n=23$ & - & $\mathrm{F}=6.895, P=0.002^{c}$ & Pro-DLB $<$ pro-AD \\
\hline \multicolumn{6}{|l|}{ Hippocampal atrophy, ${ }^{\dagger} 0 / 1 / 2 / 3 / 4$} \\
\hline Left hippocampus & $14 / 10 / 2 / 2 / 0$ & $5 / 16 / 4 / 2 / 0$ & $27 / 5 / 1 / 0 / 0$ & $H=23.992, P<0.0001^{c}$ & $\mathrm{HC}<$ pro-AD and pro-DLB \\
\hline Right Hippocampus & $14 / 10 / 4 / 0 / 0$ & $5 / 14 / 7 / 1 / 0$ & $22 / 8 / 2 / 1 / 0$ & $H=13.591, P<0.001^{c}$ & Pro- $\mathrm{AD}<\mathrm{HC}$ and pro-DLB \\
\hline
\end{tabular}

Abbreviations: $A \beta_{1-42}$ amyloid- $\beta_{1-42}$, CAF Clinician Assessment of Fluctuation, CDR Clinical Dementia Rating, ChEl cholinesterase inhibitor, CSF cerebrospinal fluid, Dopa levodopa or dopaminergic agonists, $H C$ healthy elderly control subjects, MMSE Mini Mental State Examination, NCL old age psychiatry, geriatric medicine or neurology services from Newcastle upon Tyne, NL neuroleptic, pro-AD prodromal Alzheimer's disease,

pro-DLB prodromal dementia with Lewy bodies, RBD rapid eye movement sleep behaviour disorder, SXB tertiary memory clinic of Strasbourg, TMT Trail Making Test

Tukey's post hoc test for analysis of variance (F), Mann-Whitney post hoc test in IBM SPSS software (H)

${ }^{\mathrm{b}}$ Education level: 1 = before high school, 2 = high school, 3 = university

'Statistically significant value

dpercentage of patients with test failure according to the normative data of Tombaugh, 2004 [30]

As rated on Unified Parkinson's Disease Rating Scale [28]

According to Scheltens et al. [31] 

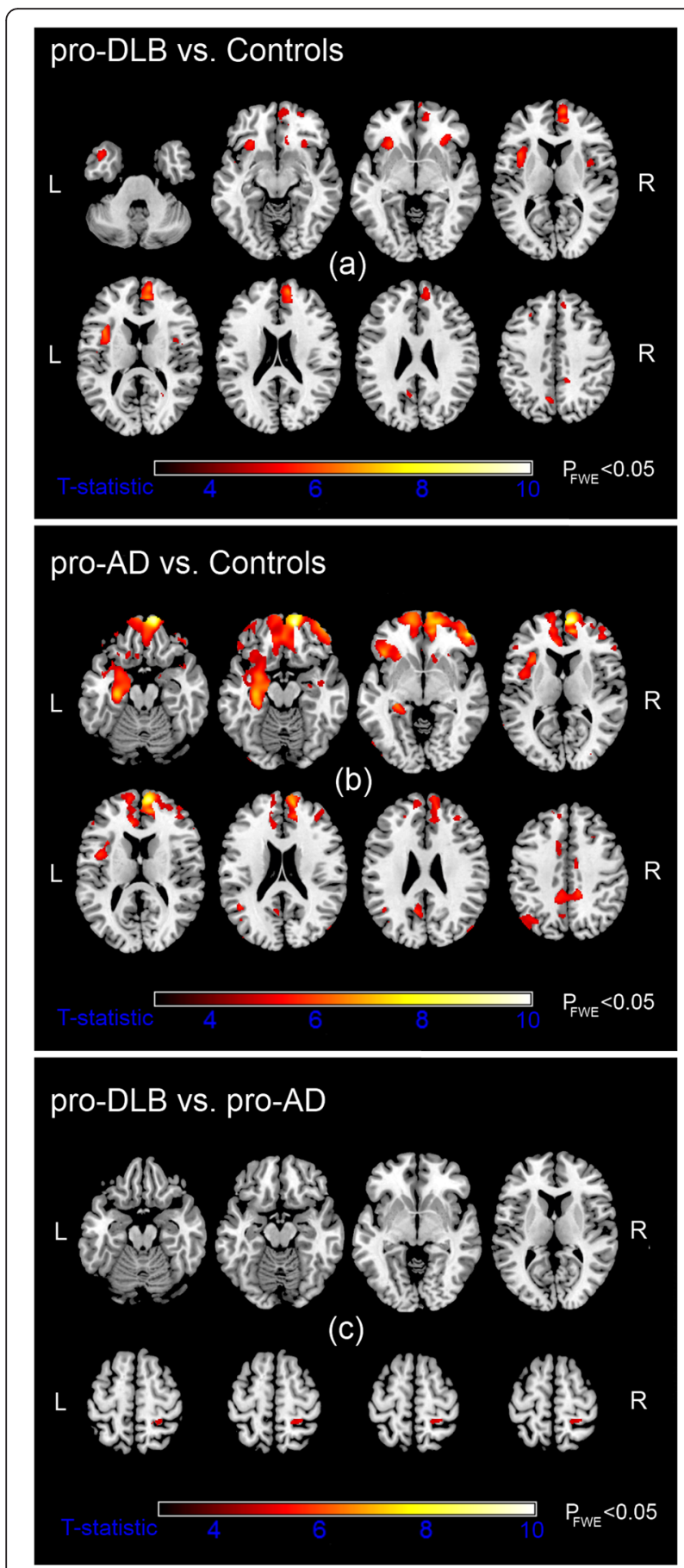

Fig. 2 Significant grey matter (GM) loss in the prodromal dementia with Lewy bodies (pro-DLB) (a) and prodromal Alzheimer's disease (pro-AD) (b) groups relative to healthy older control subjects. GM atrophy in pro-AD compared with pro-DLB (c). Results $\left(P_{\text {FWE }} \leq 0.05\right)$ are superimposed upon a magnetic resonance imaging $T 1$-weighted brain template image in axial views. $L$ left, $R$ right, FWE family-wise error

significance of this area. No significant GM loss was found in pro-DLB that was greater than that in pro-AD.
As an exploratory exercise, we also investigated patterns of GM loss between visual hallucinators and nonhallucinators in pro-DLB. Significant GM volume loss in the left cuneus was associated with the presence of visual hallucinations (Fig. 3). Table 2 presents the location and peak significance of these areas. GM atrophy in non-hallucinators did not exceed that in hallucinators.

\section{Regression analyses}

Effects of GM volume loss on parkinsonism and fluctuations were separately investigated in pro-DLB (age, sex and TIV adjusted). Associations between any of these measures and GM volume did not yield any significant results.

\section{Discussion}

We report distinct GM atrophy in pro-DLB and pro-AD compared with $\mathrm{HC}$. Compared with $\mathrm{HC}$ the group, the pro-DLB group was characterised by cortical atrophy in insulae, structures and anterior cingulate cortex. Compared with $\mathrm{HC}$, pro-AD was characterised by a widespread cortical atrophic pattern which included the hippocampi and temporal, parietal and frontal structures. The comparison of patients with pro-AD with patients with pro-DLB demonstrated more atrophy the right parietal lobe in the former than in the latter group.

The results we obtained with patients with pro-AD are in accord with the literature. The progression of atrophy with time in pro- $\mathrm{AD}$ was previously described as beginning in the medial temporal lobes [37], then extending to the parietal and finally the frontal lobes $[27,38,39]$. On one hand, the strong involvement of the frontal lobe is unusual in MCI-AD [38]. On the other hand, more impaired MCI [40] and MCI converters to AD dementia [41] have greater atrophy in the frontal lobe at the initial scan.

The atrophy found in patients with pro-DLB compared with $\mathrm{HC}$ has not been described previously, to our knowledge. Notably, we obtained similar results with the same patients on the basis VBM-DARTEL as we did with FreeSurfer methods [13], showing clear atrophic involvement of the anterior insulae in pro-DLB. Previously, we observed that cortical thinning was more apparent in the right insula, whereas in the present study, we observed GM insular atrophy bilaterally. This may reflect subtleties between MRI techniques when quantifying volumetric changes [42]. However, the discovery of the insula change in pro-DLB with two techniques in the same cohort reinforces the link between pro-DLB and early changes in the insula. This overlaps with voxel-wise meta-analytic data on cortical atrophy of patients with DLB at the stage of dementia, based on findings of bilateral insular and basal ganglia atrophy [22]. The insula is involved in integrating somatosensory, 
Table 2 Location and peak significance of grey matter volume loss using VBM-DARTEL

\begin{tabular}{|c|c|c|c|c|c|}
\hline & Voxel level $\left(P_{\mathrm{FWE}}\right)$ & Extent $(k)$ & $T, Z$ scores & MNI coordinates $(x, y, z)(\mathrm{mm})$ & Region \\
\hline \multirow[t]{13}{*}{ Pro-DLB vs. control subjects } & \multirow[t]{2}{*}{$<0.001$} & \multirow[t]{2}{*}{2361} & $7.1,6.0$ & $11,57,18$ & Right superior frontal \\
\hline & & & $7.1,6.0$ & $9,50,13$ & Right paracingulate \\
\hline & $<0.001$ & 461 & $6.8,5.8$ & $-38,11,13$ & Left insula \\
\hline & 0.001 & 1321 & $6.4,5.5$ & $-29,24,-6$ & Left insula \\
\hline & 0.001 & 340 & $6.1,5.3$ & $-8,-63,45$ & Left precuneus \\
\hline & 0.002 & 53 & $5.9,5.2$ & $14,-43,43$ & Right precuneus \\
\hline & 0.002 & 359 & $5.9,5.2$ & $30,29,-3$ & Right inferior frontal \\
\hline & 0.006 & 137 & $5.6,5.0$ & $39,5,7$ & Right insula \\
\hline & 0.009 & 35 & $5.5,4.9$ & $5,68,-3$ & Right medial frontal \\
\hline & 0.016 & 25 & $5.3,4.8$ & $-14,60,7$ & Left medial frontal \\
\hline & 0.017 & 15 & $5.3,4.8$ & $-29,29,40$ & Left middle frontal \\
\hline & 0.018 & 31 & $5.3,4.7$ & $26,56,-11$ & Right superior frontal \\
\hline & 0.033 & 12 & $5.1,4.6$ & $-14,47,0$ & Left anterior cingulate \\
\hline \multirow[t]{22}{*}{ Pro-AD vs. control subjects } & \multirow[t]{7}{*}{$<0.001$} & \multirow[t]{7}{*}{24,640} & $9.8,7.4$ & $12,59,-15$ & Right medial frontal \\
\hline & & & $8.6,6.8$ & $50,47,-3$ & Right inferior frontal \\
\hline & & & $8.0,6.5$ & $-30,-19,-18$ & Left hippocampus \\
\hline & & & $7.4,6.2$ & $42,54,-14$ & Right middle frontal \\
\hline & & & $7.3,6.1$ & $-44,26,-5$ & Left inferior frontal \\
\hline & & & $7.3,6.1$ & $-12,62,-3$ & Left medial frontal \\
\hline & & & $7.3,6.1$ & $-9,69,--9$ & Left superior frontal \\
\hline & \multirow[t]{2}{*}{$<0.001$} & \multirow[t]{2}{*}{1144} & $6.7,5.7$ & $35,21,-42$ & Right temporal pole \\
\hline & & & $6.1,5.3$ & $39,8,-33$ & Right superior temporal \\
\hline & \multirow[t]{2}{*}{0.001} & \multirow[t]{2}{*}{1881} & $6.3,5.5$ & $12,-39,42$ & Right posterior cingulate \\
\hline & & & $6.1,5.3$ & $-8,-39,40$ & Left posterior cingulate \\
\hline & 0.002 & 176 & $6.0,5.2$ & $33,-81,-53$ & Right posterior cerebellum \\
\hline & 0.003 & 67 & $5.9,5.2$ & $11,-9,39$ & Right mid-cingulate \\
\hline & 0.004 & 392 & $5.8,5.1$ & $15,24,61$ & Right superior frontal \\
\hline & 0.004 & 94 & $5.8,5.1$ & $-47,-84,-9$ & Left inferior occipital \\
\hline & 0.004 & 145 & $5.8,5.1$ & $-5,-48,-27$ & Left anterior cerebellum \\
\hline & 0.005 & 527 & $5.7,5.0$ & $-33,-70,39$ & Left precuneus \\
\hline & 0.007 & 178 & $5.6,5.0$ & $-11,15,39$ & Left mid-cingulate \\
\hline & 0.008 & 113 & $5.5,4.9$ & $48,-76,24$ & Right middle temporal \\
\hline & 0.011 & 12 & $5.4,4.8$ & $18,-72,56$ & Right superior parietal \\
\hline & 0.011 & 137 & $5.4,4.8$ & $29,-12,-14$ & Right hippocampus \\
\hline & 0.012 & 42 & $5.4,4.8$ & $-60,2,-27$ & Left middle temporal \\
\hline Pro-AD vs. pro-DLB & 0.006 & 79 & $5.6,4.9$ & $20,-42,60$ & Right superior parietal \\
\hline Pro-DLB (hallucinators vs. non-hallucinators) & $0.002^{\mathrm{a}}$ & 82 & $3.1,2.8$ & $-5,-84,18$ & Left cuneus BA17 \\
\hline
\end{tabular}

Abbreviations: BA Brodmann area, FWE family-wise error, MNI Montreal Neurological Institute, pro-AD prodromal Alzheimer's disease, pro-DLB prodromal dementia with Lewy bodies, VBM-DARTEL voxel-based morphometry with diffeomorphic anatomical registration through exponentiated lie algebra

Table depicts voxel-level significance $\left(P_{\mathrm{FWE}}\right)$, spatial extent $(k), T$ and $Z$ scores, MNI coordinates and anatomical region

ancorrected $P$ value

autonomic and cognitive-affective information to guide behaviour [43], and specifically the anterior insula has been described as part of a 'salience network' due to its consistent activation during cognitively demanding tasks. It also has been implicated in switching brain networks involved in cognition, including the central executive and default mode network [44]. The anterior insula has an abundance of specific neurons, namely the von Economo neurons (VENs), located in layer 5 of the cortex with a predominance in the right hemisphere, the same 


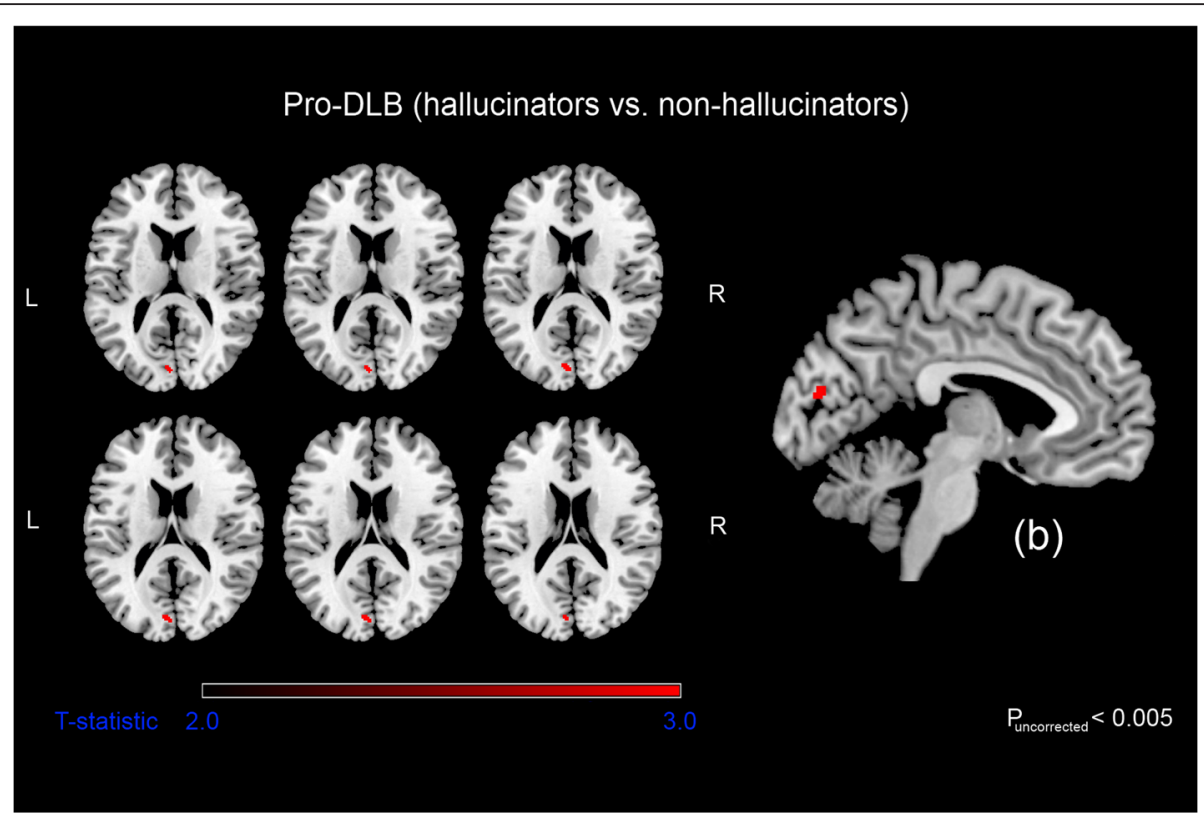

Fig. 3 Significant grey matter loss in prodromal dementia with Lewy bodies (pro-DLB) visual hallucinators compared with pro-DLB non-hallucinators. Results $\left(P_{\text {uncorrected }} \leq 0.005\right)$ superimposed on a magnetic resonance imaging T1-weighted brain template scan in axial (on the left) and sagittal (on the right, b). $L$ left, $R$ right

region we have found to be atrophic and thinner in proDLB [45]. Notably, in the right anterior cingulate cortex, an area which we found to be atrophic in this study, is also rich in VENs. Because of the larger size of VENs compared with pyramid neurons, they are purported be involved in the fast assessment of complex situations [45], as well as in the salience network function [46], and thus it might be hypothesised that deficits in this region might be pertinent to the cognitive slowing and attentional deficits which characterise DLB. This is supported by the observation that increasing functional dyssynchrony between frontal areas inclusive of the insula and parietal areas, as measured using resting state MRI, is associated with increasing cognitive fluctuations in patients with DLB [24]. Whether there is a specific vulnerability of VENs in DLB remains to be confirmed, however.

When we compared pro-DLB with pro-AD, we found subtle right parietal GM volume reductions in patients with pro-AD, and this is in line with our previous findings demonstrating cortical thinning of this region in AD compared with DLB [13]. Notably, we did not find evidence of any relative atrophy of the hippocampi in pro-AD compared with pro-DLB. However, this is consistent with our visual rating data which demonstrated that both groups had relatively mild hippocampal atrophy at the prodromal stage, with only subtle differences between the two diseases. Longitudinally, however, it might be expected that there would be an increasing divergence between pro-AD and pro-DLB cohorts, with greater hippocampal loss in the former; certainly, longitudinal comparisons in established dementia demonstrate that marked temporal and hippocampal thinning is a feature of AD but not DLB [47].

We found an association between GM loss in the left cuneus and visual hallucinations in our pro-DLB group. The cuneus is a secondary visual area (Brodmann area 18) and is of importance for recognition and extraction of object features (shape, colour, movement). Dysfunction of the cuneus is responsible for errors in visual processing [48]. The involvement of this visual area in hallucinations of patients with DLB is consistent with previous studies [48-50]. The involvement of the cuneus is congruent with the top-down/bottom-up models on hallucinations [51], since we have found a key region for visual processing (bottom-up).

Our study has some limitations. In the present study, we have used a combination of pre-existing criteria for $\mathrm{MCI}$ and DLB, but a primary issue is that operational consensus criteria for pro-DLB remain to be established. In addition, whilst patients continue to be followed longitudinally, our cohort is relatively recent in its inception, and the definite diagnostic trajectory of patients with prodromal impairment remains to be established either clinically or neuropathologically, particularly with regard to DLB. Moreover, the possibility that an incorrect classification of patients confounded the results cannot be excluded. However, we used the McKeith et al. criteria, which have an excellent specificity (>95 \%) [52, 53] compared with gold standard 
neuropathological diagnosis. In addition, we excluded other pathologies, such as psychiatric illness, other neurological diseases and co-occurrence of $\mathrm{AD}$ and DLB (see Fig. 1 flowchart). Furthermore, the majority of our patients with pro-DLB had RBD (>50\%), which enhances the specificity of the diagnosis [54]. We also systematically looked for subtle clinical symptoms such as anosmia/hyposmia, constipation and other autonomic features (data not shown) [55], as these have previously been demonstrated to improve the diagnostic specificity of patients with proDLB [55]. Finally, we used CSF analysis for most of the patients and obtained similar results for patients with $\mathrm{AD}$ and patients with DLB.

From a technical perspective, it could also be argued that a drawback of our study is the fact that data were collected from two sites with differing imaging protocols. Unfortunately, we were not able to include MRI sequences in the model, as the relative numbers in each group were disproportionately represented (i.e., $30 \mathrm{HC}$ at NCL, 3 at SXB; $1 \mathrm{AD}$ at NCL, 26 at SXB; and $2 \mathrm{DLB}$ at NCL, 26 at SXB). Therefore, it was difficult to obtain accurate parametric estimates without considerable error. However, centre effects were less likely to have influenced the between-patient contrast, as the results did not significantly differ when we examined only SXB patients in the analysis. A single-centre study should be conducted to confirm these results. Last, in a possible future analysis using resampling/subsampling techniques, the reliability of the prodromal results could be examined for validation purposes, although this would require larger cohorts.

\section{Conclusions}

Our data suggest that patients with prodromal disease have different patterns of atrophy depending on the pathology. Patients with pro-DLB seem to have more restricted areas of atrophy inclusive of the insulae and anterior cingulate cortex, and patients with pro-AD have more diffuse areas. New studies using MRI are acutely needed, particularly those considering perfusion, functional connectivity and structural connectivity, as well as other imaging modalities, such as positron emission tomography metabolic and amyloid radiotracers, to better understand the specificity of patients with pro-DLB.

\footnotetext{
Abbreviations

$A \beta_{1-42}$, amyloid- $\beta_{1-42} ; A D$, Alzheimer's disease; ANOVA, analysis of variance; $B A$, Brodmann area; CAF, Clinician Assessment of Fluctuation; CDR, Clinical Dementia Rating; ChEl, cholinesterase inhibitor; CSF, cerebrospinal fluid; 3D, three-dimensional; DARTEL, diffeomorphic anatomical registration through exponentiated lie algebra; DLB, dementia with Lewy bodies; Dopa, levodopa or dopaminergic agonists; DSM-V, Diagnostic and Statistical Manual of Mental Disorders, Fifth Edition; FWE, family-wise error; GM, grey matter; HC, healthy elderly control subjects; $\mathrm{MCl}$, mild cognitive impairment; MMSE, Mini Mental State Examination; MNI, Montreal Neurological Institute; MRI, magnetic resonance imaging; $\mathrm{NCL}$, old age psychiatry, geriatric medicine or neurology
}

services from Newcastle upon Tyne; NL, neuroleptic; pro-AD, prodromal Alzheimer's disease; pro-DLB, prodromal dementia with Lewy bodies; RBD, rapid eye movement sleep behaviour disorder; SXB, tertiary memory clinic of Strasbourg; TE, echo time; TIV, total intracranial volume; TMT, Trail Making Test; TR, repetition time; UPDRS, Unified Parkinson's Disease Rating Scale; VBM, voxel-based morphometry; VEN, von Economo neuron; WM, white matter

\begin{abstract}
Acknowledgements
The authors thank Mélanie Stackfleth, Heather Hunt, Barbara Jung and Laetitia Berly for help with organisation; Mathias Bilger, Laure Di Bitonto, Emmanuelle Ehrhard, Jennifer Kemp, Alison Killen, Nicola Barnett, Catherine Kleitz, Nadine Longato, Laetitia Monjoin and Natacha Vogt for conducting neuropsychological assessments; and Corinne Marrer, Jean-Paul Armspach, Camille Heitz, Stéphane Kremer, Fabrice Bing and Daniel Gounot for performing MRI
\end{abstract}

\section{Funding}

This study was funded by Appel à Projet Interne (API) of the University Hospital of Strasbourg, Alsace Alzheimer 67, Fondation Université de Strasbourg and famille Jean Amrhein, and Projet Hospitalier de Recherche Clinique (PHRC) inter-régional (IDRCB 2012-A00992-41). The work was also supported by the following: the Newcastle Healthcare Charity (BH0070250): Academy of Medical Sciences, Wellcome Trust Starter Grants scheme for Clinical Lecturers (BH090112 to JPT); Wellcome Intermediate Clinical Fellowship (BH083281 to JPT); National Institute for Health Research (NIHR) Newcastle Biomedical Research Centre in Ageing and Chronic Disease and Biomedical Research Unit in Lewy Body Dementia, based at Newcastle upon Tyne Hospitals NHS Foundation Trust and Newcastle University; NIHR Dementia Biomedical Research Unit at Cambridge University Hospitals NHS Foundation Trust and the University of Cambridge. The views expressed are those of the authors and not necessarily those of the NHS, the NIHR or the Department of Health.

\section{Authors' contributions}

SJC performed the image processing and revised the manuscript. BC, NP, $C M H, C D, J T O ' B$ and IM examined patients, diagnosed patients with DLB and $A D$, participated in the design of the study and revised the manuscript. PLdS performed image acquisition and MRI sequence implementation and revised the manuscript. JPT conceived of the study, participated in its design and coordination, and revised the manuscript. FB conceived of the study, participated in its design and coordination, examined patients, diagnosed patients with $D L B$ and $A D$, analysed patients' records, performed the statistical analysis and drafted the manuscript. All authors read and approved the final manuscript.

\section{Competing interests}

FB has received speaker's honoraria and travel expenses from Roche, Biogen Idec, Novartis and Merck Serono.

SJC, CD, CMH, BC and PLdS declare that they have no competing interests. JTO'B has acted as a consultant for GE Healthcare, Avid

Radiopharmaceuticals/Lilly, Alliance Medical, Piramal, TauRx and Accera and has received grant funding from Avid Radiopharmaceuticals.

NP has received travel expenses from Novartis and Lundbeck. IM has served on scientific advisory board for GE Healthcare (GEHC) and Nutricia. He has received speaker's honoraria from GEHC.

JPT serves on the Specialist Advisory Committee for the Lewy Body Society. He has received speaker's honoraria and travel expenses from GE Healthcare and MentisCura. He was a consultant for Novartis. He has received research support from National Institute for Health Research (NIHR) Biomedical Research Unit (BRU) and Biomedical Research Centre (BRC) funding, and from Newcastle Healthcare Charity (BH0070250), Academy of Medical Sciences, Wellcome Trust Starter Grants Scheme for Clinical Lecturers (BH090112), and a Wellcome Intermediate Clinical Fellowship (BH083281). No non-financial conflicts of interest exist for any of the authors.

This study is not industry-sponsored.

\section{Ethics approval and consent to participate}

This research was approved by the local ethics committees at SXB (Comité de Protection des Personnes Est IV) and NCL (NRES Committee North East Sunderland and NRES Committee North East Newcastle \& North Tyneside 2). 
All subjects or, where appropriate, their nearest relative provided written informed consent to participate.

\begin{abstract}
Author details
${ }^{1}$ Geriatrics day hospital and neuropsychology unit. Geriatrics department and Neurology service, Memory Resources and Research Centre (CMRR), University Hospital of Strasbourg, Strasbourg, France. ${ }^{2}$ Team IMIS/ Neurocrypto, French National Center for Scientific Research (CNRS), ICube Laboratory and Fédération de Médecine Translationnelle de Strasbourg (FMTS), University of Strasbourg, Strasbourg, France. ${ }^{3}$ Institute of Neuroscience, Campus for Aging and Vitality, Newcastle University, Newcastle upon Tyne, UK. ${ }^{4}$ Department of Psychiatry, University of Cambridge, Cambridge Biomedical Campus, Cambridge, UK.
\end{abstract}

Received: 14 November 2015 Accepted: 29 June 2016 Published online: 20 July 2016

\section{References}

1. McKeith IG, Dickson DW, Lowe J, Emre M, O'Brien JT, Feldman H, et al. Diagnosis and management of dementia with Lewy bodies: third report of the DLB Consortium. Neurology. 2005;65(12):1863-72. doi:10.1212/01.wnl. 0000187889.17253.b1.

2. American Psychiatric Association. Diagnostic and statistical manual of mental disorders. 5th ed. Washington, DC: American Psychiatric Publishing; 2013.

3. Molano J, Boeve B, Ferman T, Smith G, Parisi J, Dickson D, et al. Mild cognitive impairment associated with limbic and neocortical Lewy body disease: a clinicopathological study. Brain. 2010;133(Pt 2):540-56. doi:10.1093/brain/awp280. PubMed PMID: 19889717, PubMed Central PMCID: PMC2822633.

4. Yoshizawa H, Vonsattel JP, Honig LS. Early neuropsychological discriminants for Lewy body disease: an autopsy series. J Neurol Neurosurg Psychiatry. 2013;84(12):1326-30. doi:10.1136/jnnp-2012-304381.

5. Ferman TJ, Smith GE, Kantarci K, Boeve BF, Pankratz VS, Dickson DW, et al. Nonamnestic mild cognitive impairment progresses to dementia with Lewy bodies. Neurology. 2013;81(23):2032-8. doi:10.1212/01.wnl.0000436942. 55281.47. PubMed PMID: 24212390, PubMed Central PMCID: PMC3854825.

6. Petrova M, Pavlova R, Zhelev Y, Mehrabian S, Raycheva M, Traykov L. Investigation of neuropsychological characteristics of very mild and mild dementia with Lewy bodies. J Clin Exp Neuropsychol. 2016;38(3):354-60. doi:10.1080/13803395.2015.1117058.

7. Mondon K, Gochard A, Marqué A, Armand A, Beauchamp D, Prunier C, et al. Visual recognition memory differentiates dementia with Lewy bodies and Parkinson's disease dementia. J Neurol Neurosurg Psychiatry. 2007;78(7): 738-41. doi:10.1136/jnnp.2006.104257.

8. Breitve MH, Hynninen MJ, Brønnick K, Chwiszczuk L, Auestad BH, Aarsland $D$, et al. A longitudinal study of anxiety and cognitive decline in dementia with Lewy bodies and Alzheimer's disease. Alzheimers Res Ther. 2016;8:3. doi:10.1186/s13195-016-0171-4.

9. Falkowski J, Hynan LS, Womack KB, Hatanpaa KJ, White CL, Weiner MF. Does past or present depression differentiate Lewy body from Alzheimer disease? Int Psychogeriatr. 2015;27(4):693-4. doi:10.1017/S1041610214001793.

10. Aarsland D, Mosimann UP, McKeith IG. Role of cholinesterase inhibitors in Parkinson's disease and dementia with Lewy bodies. J Geriatr Psychiatry Neurol. 2004;17(3):164-71.

11. Oesterhus R, Soennesyn H, Rongve A, Ballard C, Aarsland D, Vossius C. Longterm mortality in a cohort of home-dwelling elderly with mild Alzheimer's disease and Lewy body dementia. Dement Geriatr Cogn Disord. 2014;38(3-4):161-9. doi:10.1159/000358051.

12. Donaghy P, McKeith I. The clinical characteristics of dementia with Lewy bodies and a consideration of prodromal diagnosis. Alzheimers Res Ther. 2014;6(4):46. doi:10.1186/alzrt274.

13. Blanc F, Colloby SJ, Philippi N, de Pétigny $X$, Jung B, Demuynck C, et al. Cortical thickness in dementia with Lewy bodies and Alzheimer's disease: a comparison of prodromal and dementia stages. PLoS One. 2015;10(6): e0127396. doi:10.1371/journal.pone.0127396.

14. Boeve BF. Mild cognitive impairment associated with underlying Alzheimer's disease versus Lewy body disease. Parkinsonism Relat Disord. 2012;18 Suppl 1:S41-4. doi:10.1016/S1353-8020(11)70015-3.

15. Testa C, Laakso MP, Sabattoli F, Rossi R, Beltramello A, Soininen H, et al. A comparison between the accuracy of voxel-based morphometry and hippocampal volumetry in Alzheimer's disease. J Magn Reson Imaging. 2004;19(3):274-82. doi:10.1002/jmri.20001.

16. Mak HK, Zhang Z, Yau KK, Zhang L, Chan Q, Chu LW. Efficacy of voxel-based morphometry with DARTEL and standard registration as imaging biomarkers in Alzheimer's disease patients and cognitively normal older adults at 3.0 Tesla MR imaging. J Alzheimers Dis. 2011;23(4):655-64. doi:10.3233/JAD-2010-101659.

17. Bergouignan $L$, Chupin $M$, Czechowska $Y$, Kinkingnéhun $S$, Lemogne $C$, Le Bastard G, et al. Can voxel based morphometry, manual segmentation and automated segmentation equally detect hippocampal volume differences in acute depression? Neuroimage. 2009;45(1):29-37. doi:10.1016/ j.neuroimage.2008.11.006.

18. Kakeda S, Korogi Y. The efficacy of a voxel-based morphometry on the analysis of imaging in schizophrenia, temporal lobe epilepsy, and Alzheimer's disease/mild cognitive impairment: a review. Neuroradiology. 2010;52(8):711-21. doi:10.1007/s00234-010-0717-2.

19. Weintraub D, Dietz N, Duda JE, Wolk DA, Doshi J, Xie SX, et al. Alzheimer's disease pattern of brain atrophy predicts cognitive decline in Parkinson's disease. Brain. 2012;135(1):170-80. doi:10.1093/brain/awr277.

20. Burton EJ, Karas G, Paling SM, Barber R, Williams ED, Ballard CG, et al. Patterns of cerebral atrophy in dementia with Lewy bodies using voxelbased morphometry. Neuroimage. 2002;17(2):618-30.

21. Whitwell JL, Weigand SD, Shiung MM, Boeve BF, Ferman TJ, Smith GE, et al. Focal atrophy in dementia with Lewy bodies on MRI: a distinct pattern from Alzheimer's disease. Brain. 2007;130(Pt 3):708-19. doi:10.1093/brain/awl388. PubMed PMID: 17267521, PubMed Central PMCID: PMC2730778.

22. Zhong J, Pan P, Dai Z, Shi H. Voxelwise meta-analysis of gray matter abnormalities in dementia with Lewy bodies. Eur J Radiol. 2014;83(10):18704. doi:10.1016/j.ejrad.2014.06.014.

23. Chiba Y, Fujishiro H, Iseki E, Ota K, Kasanuki K, Hirayasu Y, et al. Retrospective survey of prodromal symptoms in dementia with Lewy bodies: comparison with Alzheimer's disease. Dement Geriatr Cogn Disord. 2012;33(4):273-81. doi:10.1159/000339363.

24. Peraza LR, Kaiser M, Firbank M, Graziadio S, Bonanni L, Onofrj M, et al. fMR resting state networks and their association with cognitive fluctuations in dementia with Lewy bodies. Neuroimage Clin. 2014:4:558-65. doi:10.1016/j. nicl.2014.03.013. PubMed PMID: 24818081; PubMed Central PMCID: PMCPmc3984441.

25. Blanc F, Noblet V, Philippi N, Cretin B, Foucher J, Armspach JP, et al. Right anterior insula: core region of hallucinations in cognitive neurodegenerative diseases. PLoS One. 2014;9(12):e114774. doi:10.1371/journal.pone.0114774.

26. Greenfield JG, Bosanquet FD. The brain-stem lesions in parkinsonism. J Neurol Neurosurg Psychiatry. 1953;16(4):213-26.

27. Whitwell JL, Przybelski SA, Weigand SD, Knopman DS, Boeve BF, Petersen $R C$, et al. 3D maps from multiple MRI illustrate changing atrophy patterns as subjects progress from mild cognitive impairment to Alzheimer's disease. Brain. 2007:130(Pt 7):1777-86. doi:10.1093/brain/awm112. PubMed PMID: 17533169, PubMed Central PMCID: PMC2752411.

28. Fahn S, Elton R, Members of the UPDRS Development Committee. Unified Parkinson's Disease Rating Scale. In: Fahn S, Marsden CD, Calne DB, Goldstein M, editors. Recent developments in Parkinson's disease, vol. 2. Florham Park, NJ: Macmillan Health Care Information; 1987. p. 153-64.

29. Walker MP, Ayre GA, Cummings JL, Wesnes K, McKeith IG, O'Brien JT, et al. The Clinician Assessment of Fluctuation and the One Day Fluctuation Assessment Scale: two methods to assess fluctuating confusion in dementia. Br J Psychiatry. 2000;177:252-6.

30. Tombaugh TN. Trail Making Test A and B: normative data stratified by age and education. Arch Clin Neuropsychol. 2004;19(2):203-14. doi:10.1016/ S0887-6177(03)00039-8.

31. Scheltens P, Leys D, Barkhof F, Huglo D, Weinstein HC, Vermersch P, et al. Atrophy of medial temporal lobes on MRI in "probable" Alzheimers disease and normal ageing: diagnostic value and neuropsychological correlates. J Neurol Neurosurg Psychiatry. 1992;55(10):967-72.

32. Dubois B, Feldman HH, Jacova C, DeKosky ST, Barberger-Gateau P, Cummings J, et al. Research criteria for the diagnosis of Alzheimer's disease: revising the NINCDS-ADRDA criteria. Lancet Neurol. 2007;6(8):734-46. doi:10.1016/S1474-4422(07)70178-3.

33. Petersen RC. Mild cognitive impairment as a diagnostic entity. J Int Med. 2004;256(3):183-94. doi:10.1111/j.1365-2796.2004.01388.x.

34. Ashburner J, Friston KJ. Unified segmentation. Neuroimage. 2005;26(3):83951. doi:10.1016/j.neuroimage.2005.02.018. 
35. Ashburner J. A fast diffeomorphic image registration algorithm. Neuroimage. 2007;38(1):95-113. doi:10.1016/j.neuroimage.2007.07.007.

36. Friston KJ, Worsley KJ, Frackowiak RSJ, Mazziotta JC, Evans AC. Assessing the significance of focal activations using their spatial extent. Hum Brain Mapp. 1994;1(1):214-20.

37. Fleisher AS, Sun S, Taylor C, Ward CP, Gamst AC, Petersen RC, et al. Volumetric MRI vs clinical predictors of Alzheimer disease in mild cognitive impairment. Neurology. 2008;70(3):191-9. doi:10.1212/01.wnl.0000287091. 57376.65

38. Whitwell JL, Shiung MM, Przybelski SA, Weigand SD, Knopman DS, Boeve $B F$, et al. MRI patterns of atrophy associated with progression to AD in amnestic mild cognitive impairment. Neurology. 2008;70(7):512-20. doi:10.1212/01.wnl.0000280575.77437.a2. PubMed PMID: 17898323, PubMed Central PMCID: PMC2734138.

39. Bakkour A, Morris JC, Dickerson BC. The cortical signature of prodromal AD: regional thinning predicts mild AD dementia. Neurology. 2009;72(12):104855. doi:10.1212/01.wnl.0000340981.97664.2f. PubMed PMID: 19109536, PubMed Central PMCID: PMC2677470.

40. McDonald CR, McEvoy LK, Gharapetian L, Fennema-Notestine C, Hagler DJ, Holland D, et al. Regional rates of neocortical atrophy from normal aging to early Alzheimer disease. Neurology. 2009;73(6):457-65. doi:10.1212/WNL. Ob013e3181b16431.

41. Risacher SL, Saykin AJ, West JD, Shen L, Firpi HA, McDonald BC, Alzheimer's Disease Neuroimaging Initiative (ADNI). Baseline MRI predictors of conversion from $\mathrm{MCl}$ to probable $\mathrm{AD}$ in the $\mathrm{ADNI}$ cohort. Curr Alzheimer Res. 2009;6(4):347-61. doi:10.2174/156720509788929273.

42. Clerx L, Jacobs HI, Burgmans S, Gronenschild EH, Uylings HB, Echavarri C, et al. Sensitivity of different MRI-techniques to assess gray matter atrophy patterns in Alzheimer's disease is region-specific. Curr Alzheimer Res. 2013;10(9):940-51.

43. Christopher L, Koshimori $Y$, Lang AE, Criaud M, Strafella AP. Uncovering the role of the insula in non-motor symptoms of Parkinson's disease. Brain. 2014;137(Pt 8):2143-54. doi:10.1093/brain/awu084.

44. Seeley WW, Menon V, Schatzberg AF, Keller J, Glover GH, Kenna H, et al. Dissociable intrinsic connectivity networks for salience processing and executive control. J Neurosci. 2007;27(9):2349-56. doi:10.1523/JNEUROSCI. 5587-06.2007. PubMed PMID: 17329432, PubMed Central PMCID: PMC2680293.

45. Allman JM, Watson KK, Tetreault NA, Hakeem AY. Intuition and autism: a possible role for Von Economo neurons. Trends Cogn Sci. 2005;9(8):367-73. doi:10.1016/j.tics.2005.06.008.

46. Cauda F, Torta DM, Sacco K, D'Agata F, Geda E, Duca S, et al. Functional anatomy of cortical areas characterized by Von Economo neurons. Brain Struct Funct. 2013;218(1):1-20. doi:10.1007/s00429-012-0382-9.

47. Mak E, Su L, Williams GB, Watson R, Firbank MJ, Blamire AM, et al. Progressive cortical thinning and subcortical atrophy in dementia with Lewy bodies and Alzheimer's disease. Neurobiol Aging. 2015;36(4):1743-50. doi:10.1016/j.neurobiolaging.2014.12.038.

48. Heitz C, Noblet V, Cretin B, Philippi N, Kremer L, Stackfleth M, et al. Neural correlates of visual hallucinations in dementia with Lewy bodies. Alzheimers Res Ther. 2015;7(1):6. doi:10.1186/s13195-014-0091-0.

49. Taylor J, Firbank M, He J, Barnett N, Pearce S, Livingstone A. Visual cortex in dementia with Lewy bodies: magnetic resonance imaging study. $\mathrm{Br} J$ Psychiatry. 2012;200:491-8. doi:10.1192/bjp.bp.111.099432.

50. Nagahama Y, Okina T, Suzuki N, Matsuda M. Neural correlates of psychotic symptoms in dementia with Lewy bodies. Brain. 2010;133:557-67. doi:10.1093/brain/awp295.

51. Collerton D, Perry E, McKeith I. Why people see things that are not there: a novel Perception and Attention Deficit model for recurrent complex visual hallucinations. Behav Brain Sci. 2005;28:737-94.

52. Nelson PT, Jicha GA, Kryscio RJ, Abner EL, Schmitt FA, Cooper G, et al. Low sensitivity in clinical diagnoses of dementia with Lewy bodies. J Neurol. 2010;257(3):359-66. doi:10.1007/s00415-009-5324-y. PubMed PMID: 19795154, PubMed Central PMCID: PMC2839040.

53. McKeith IG, Ballard CG, Perry RH, Ince PG, O'Brien JT, Neill D, et al. Prospective validation of consensus criteria for the diagnosis of dementia with Lewy bodies. Neurology. 2000;54(5):1050-8.
54. Ferman TJ, Boeve BF, Smith GE, Lin SC, Silber MH, Pedraza O, et al. Inclusion of RBD improves the diagnostic classification of dementia with Lewy bodies. Neurology. 2011;77(9):875-82. doi:10.1212/WNL.0b013e31822c9148. PubMed PMID: 21849645, PubMed Central PMCID: PMC3162640.

55. Fujishiro H, Iseki E, Nakamura S, Kasanuki K, Chiba Y, Ota K, et al. Dementia with Lewy bodies: early diagnostic challenges. Psychogeriatrics. 2013;13(2): 128-38. doi:10.1111/psyg.12005.

\section{Submit your next manuscript to BioMed Central and we will help you at every step:}

- We accept pre-submission inquiries

- Our selector tool helps you to find the most relevant journal

- We provide round the clock customer support

- Convenient online submission

- Thorough peer review

- Inclusion in PubMed and all major indexing services

- Maximum visibility for your research

Submit your manuscript at www.biomedcentral.com/submit
Ciomed Central 